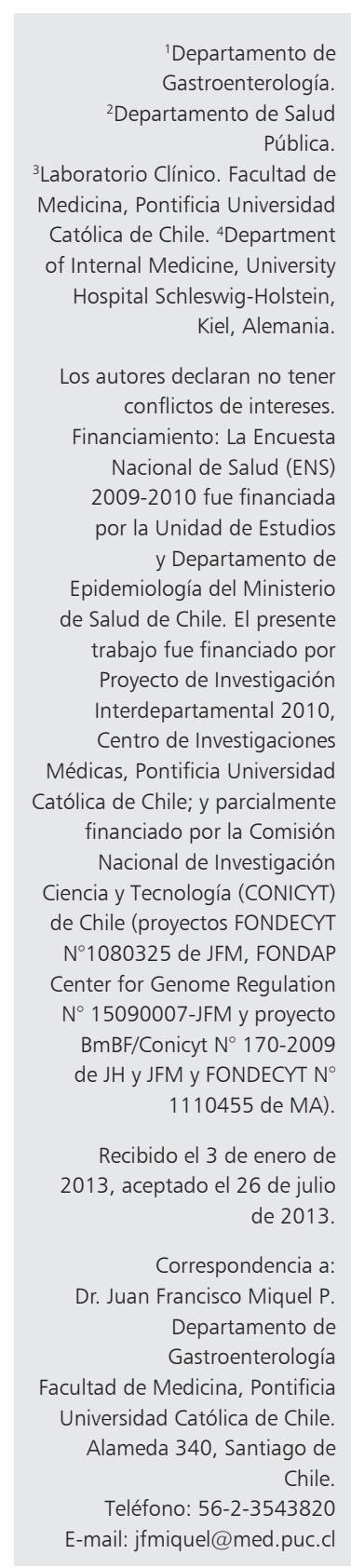

\section{Prevalencia de síndrome de Gilbert y sus determinantes genéticas en población chilena}

\author{
LUIS MÉNDEZ1 ${ }^{1}$, MARCELA LAGOS ${ }^{3}$, TERESA QUIROGA ${ }^{3}$, \\ PAULA MARGOZZINI ${ }^{2}$, LORENA AZÓCAR ${ }^{1}$, HÉCTOR R. MOLINA ${ }^{1}$, \\ ALEJANDRA VERA ${ }^{3}$, LUIS VILLARROEL ${ }^{2}$, MARCO ARRESE $^{1}$, \\ JOCHEN HAMPE ${ }^{4}$, STEPHAN BUCH ${ }^{4}$, JUAN F. MIQUEL ${ }^{1}$
}

\section{Prevalence of Gilbert syndrome and its genetic determinants in Chile}

Background: In Europeans the TATA box TA7 repeat promoter variant in the UGT1A1 gene (UGT1A1*28) is the major determinant of bilirubin levels. Aim: To study the prevalence of Gilbert Syndrome (GS) and its genetic determinants in Chile. Material and Methods: Three different studies were conducted. The prevalence of GS in Chile was assessed in 991 subjects with normal liver tests (ALT and GGT) from the $2^{\text {nd }}$ National Health Survey. We defined GS as a total bilirubin (TB) between 1.4-5mg/dL. The second study assessed the genotype prevalence of SNP rs6742078 (in LD with UGT1A1*28) and rs4149056 in 500 DNA samples of non-related Hispanics. Finally, a case-control study was designed to assess the phenotype-genotype correlation. UGT1A1*28 and rs4149056 variants were determined by direct sequencing and allelic discrimination assays (TaqMan), respectively. Results: Prevalence of GS in the general Chilean population was $2.6 \%$ (4.5\% in males and $0.5 \%$ in female). No correlation with age, educational level or home location was found. Genotypes for UGT1A1*28 (TA6/6 50.5\%, TA6/7 $37.8 \%$, TA7/7 11.7\%) and rs4149056 (TT 74.1\%, CT 22.8\%, and CC 3.1\%) variants were similar to Europeans. In the case-control study, most patients with GS were homozygotes for UGT1A1*28 (TA7/7, 74\%). Of note, 44\% of patients with intermediate TB levels were also TA7/7, compared to 7\% in normal subjects. SLCO1B1 genotype was not correlated with TB levels. Conclusions: While the prevalence of GS was lower in Chile compared to Europeans ( $5 \%)$, the prevalence of UGT1A1*28 homozygotes was similar ( 12\%). In Chilean Hispanics, the UGT1A1*28 variant explain $75 \%$ of GS phenotype.

(Rev Med Chile 2013; 141: 1266-1274).

Key words: Gilbert disease; Glucuronosyl transferase; Hyperbilirubinemia; Organic anion transporters.
E 1 síndrome de Gilbert (SG) se define como una elevación discreta de los niveles de bilirrubina sérica no conjugada en ausencia de hemólisis o enfermedad hepática y es la principal causa de hiperbilirrubinemia en población gene$\mathrm{ral}^{1}$. Fue descrito por A. Gilbert ${ }^{2}$ como una "ictericia simple familiar” y luego por Meulengracht como "icterus intermittens juvenilis", aludiendo a su carácter benigno y fluctuante. Tradicionalmente el diagnóstico de SG se considera una condición benigna sin relevancia clínica. Diferentes estudios han reportado prevalencias poblacionales de SG entre $2,4 \%$ hasta $7 \%{ }^{4,5}$.

El descubrimiento de las bases moleculares del 
SG y el desarrollo de la farmacogenética permiten abordar este síndrome bajo nuevas perspectivas ${ }^{6}$.

La bilirrubina es una molécula hidrofóbica derivada del catabolismo de hemoglobina, mioglobina y citocromos ${ }^{1,6}$. Las enzimas responsables del catabolismo del grupo heme son heme-oxidasa (OH-1), biliverdina reductasa (BLVR) y glucoronosiltransferasa (UGT1A1). Normalmente la bilirrubina no conjugada representa $96 \%$ de la bilirrubina circulante. La bilirrubina ingresa al hepatocito por transporte facilitado, mediado por OATP1B $11^{1,6}$ y una fracción pudiera ingresar por difusión pasiva ${ }^{7}$. En el microsoma es conjugada con ácido glucorónico por la enzima UGT1A1, confiriendo mayor capacidad de transporte para su excreción al canalículo biliar mediante el transportador ABCC2 (MRP2) ${ }^{1,8}$. Los niveles de bilirrubina sérica son modulados por factores como el ayuno, estrés, infecciones, ejercicio y ciertos fármacos ${ }^{1}$. Además, las mujeres tienen menores niveles de bilirrubina que los hombres, lo que se atribuye a mayor conjugación por efecto de las hormonas sexuales ${ }^{9}$. Se considera $1 \mathrm{mg} / \mathrm{dL}$ de bilirrubina total como el límite superior normal, aunque este valor es aún debatido ${ }^{1,5,10}$.

En sujetos con SG existe una menor capacidad de glucoronización hepática de bilirrubina ${ }^{11,12}$. En 1995, Bosma y cols ${ }^{13}$ identificaron las bases genéticas del SG. Mediante secuenciación del gen UGT1A1 y su zona promotora, descubrieron una variante genética asociada al fenotipo. En sujetos caucásicos sanos, el TATA box de la región promotora del gen UGT1A1 contiene una secuencia repetitiva de seis dinucleótidos TA (TA6), en tanto los individuos con SG resultaron homocigotos para un dinucleótido adicional (TA7/TA7). Esta variante se hereda en forma autosómica recesiva con penetrancia incompleta ${ }^{14}$ y genera menores niveles transcripcionales de la enzima. Es relevante destacar que algunos xenobióticos comparten la misma vía de conjugación y excreción, por lo que el SG puede ser un predictor de toxicidad a ciertos fármacos ${ }^{6,15,16}$.

Estudios de screening genómico total (GWAS) en poblaciones europeas han confirmado que la variabilidad de los niveles de BT es explicada por la varianteTA6/TA7del genUGT1A1 y una segunda variante en el gen SLCO1B1 (rs4149056, T > C), que codifica para el transportador de aniones OATP1B1. Este transportador tiene afinidad por diferentes sustratos, entre ellos bilirrubina y esta- tinas ${ }^{17}$. La variante $\mathrm{C}$ confiere riesgo de miopatía por estatinas ${ }^{18}$.

El objetivo del presente trabajo fue estimar la prevalencia fenotípica del SG en población adulta general chilena y definir si las variantes genéticas que determinan SG genotípico en poblaciones europeas, son responsables del desarrollo del fenotipo SG en nuestra población.

\section{Pacientes y Métodos}

Se realizaron 3 estudios en poblaciones diferentes.

\section{Prevalencia poblacional del síndrome de Gilbert}

Se empleó la base de datos y sueros de la segunda Encuesta Nacional de Salud (ENS2009-2010) 19,20 (para detalles ver http://www.minsal.gob.cl/portal/ url/item/bcb03d7bc28b64dfe040010165012d23. pdf). La ENS fue un estudio realizado en hogares con una muestra nacional, probabilística, estratificada y multietápica de 5.412 personas de 15 y más años. La muestra tiene un error de estimación que no supera $20 \%$ relativo para prevalencias superiores a $4 \%$. El diseño fue aprobado por el Comité de Ética de la Pontificia Universidad Católica de Chile (PUC) y el Ministerio de Salud.

Las muestras de sangre fueron transportadas a $4^{\circ} \mathrm{C}$ a los hospitales regionales donde fueron procesadas y los sueros congelados a $-20^{\circ} \mathrm{C}$ y enviados con cadena de frío a Santiago para su análisis y almacenamiento a $-20^{\circ} \mathrm{C}$ en el laboratorio de la PUC. Se obtuvo muestra de sangre de $95 \%$ de los participantes. Se determinó niveles séricos de transaminasas (ALT) y gamma glutamil transpeptidasa (GGT) en una sub-muestra aleatoria de 2.794 sujetos. Para la determinación de bilirrubina sérica, se seleccionó en forma aleatoria 1.400 sujetos con ALT y GGT normales. El tamaño muestral fue estimado considerando un error absoluto de $0,9 \%$ (rango $4,1-5,7$ ) y un intervalo de confianza de $95 \%$. Definimos SG con BT $\geq 1,4 \mathrm{mg} / \mathrm{dl}^{5}$. Las determinaciones bioquímicas se realizaron en un equipo Roche/Hitachi (Modular P800 Chemistry Analyzer, Tokio).

\section{Prevalencia en población chilena de las variantes UGT1A $1^{\star} 28$ y SLCO1B1 c. T $>C$}

En colaboración con la Universidad de Kiel (Alemania) se genotipificaron muestras de ADN 
del Departamento de Gastroenterología-PUC, obtenidas en un estudio de poblacional de la comuna de la Florida, Santiago ${ }^{21}$ y ha sido validada para análisis de determinantes genéticas en estudios previos $^{22}$. Para calcular tamaño muestral se estimó a priori una frecuencia de $15 \%$ de homocigotos para el alelo UGT1A $1{ }^{\star} 28$ y de $2 \%$ para el genotipo infrecuente C/C SLCO1B1 (rs4149056). Considerando $20 \%$ de error relativo, se requería de $\geq 282$ sujetos para un intervalo de confianza de $95 \%$.

Se genotipificaron 500 muestras no relacionadas seleccionadas aleatoriamente de un universo de 1.100. La presencia del alelo UGT1A1 ${ }^{\star} 28$ (TA7) se determinó indirectamente analizando la variante rs6742078 (G > T) ubicada en el intrón 1 del gen UGT1A1, que se encuentra en fuerte desequilibrio de unión $\left(\mathrm{r}^{2}=0,88\right)$ con las variantes TA6/TA7 ${ }^{17}$. Se empleó el test de discriminación alélica por RTPCR con reactivos Taqman (Applied Biosystems).

\section{Estudio de correlación genotipo-fenotipo}

Se efectuó con muestras residuales de sangre de pacientes ambulatorios que acudieron entre diciembre de 2010 y marzo de 2012 a realizarse hemograma y perfil bioquímico (Laboratorio Central PUC). Se seleccionó 300 muestras de sujetos entre 18 y 35 años, agrupados de acuerdo a sus niveles de bilirrubina total: Normal (BT $\leq 1 \mathrm{mg} / \mathrm{dL}$ ), Intermedio (BT $>1 \mathrm{y}<1,4 \mathrm{mg} /$ dL) y $S G(B T \geq 1,4 \mathrm{mg} / \mathrm{dL})$. Para cada grupo se recopilaron 100 muestras. Se excluyeron casos de anemia (hemoglobina $<12$ o hematocrito $<35 \%$ ), probable hemólisis (LDH elevada y/o esquistocitos) y elevación de fosfatasas alcalinas o ALT. Luego de anonimizar las muestras, se extrajo ADN. La variante UGT1a1²8 se determinó por secuenciación directa con técnica de Sanger y test de discriminación alélica para la variante rs6742078 para evaluar el grado de desequilibrio de unión (LD) entre ambas. La variante rs4149056 se analizó mediante test de discriminación alélica. El protocolo fue aprobado por el Comité de Ética de la Facultad de Medicina de la PUC.

\section{Análisis estadístico}

Los datos se analizaron en SPSS v.17. Para las comparaciones de prevalencia entre poblaciones se usó el test exacto de Fisher, considerando un valor $\mathrm{p}<0,05$ estadísticamente significativo. $\mathrm{La}$ distribución normal se determinó con el test de Kolmogorov-Smirnov. Los casos sin distribución normal se analizaron con test no paramétricos (Mann-Whitney). Para normalizar los valores de bilirrubina de la ENS 2009-2010, los datos crudos se transformaron a logaritmo y se exploró las correlaciones con variables continuas. Las variables discretas se asociaron con el diagnóstico de SG mediante test de $\chi^{2}$. Las tasas de prevalencia y medias se calcularon utilizando los factores de expansión conforme al diseño complejo y ajustando la muestra a la demografía chilena a junio de 2010. El error estándar y los intervalos de $95 \%$ de confianza se calcularon utilizando linearización de Taylor. Para los análisis de asociaciones genotipo-fenotipo se utilizaron tablas de contingencia con test exacto de Fisher y test de $\chi^{2}$ para independencia. Para el análisis de niveles de bilirrubina en función de genotipos UGT1A1, se aplicó análisis de varianza en una vía y test de comparación múltiple de Bonferroni.

\section{Resultados}

\section{Prevalencia del síndrome de Gilbert en población general}

Del universo de sujetos de la ENS con determinaciones de ALT y GGT (2.794), se excluyó 12,4\% y $11,6 \%$ por presentar elevación de GGT y ALT, respectivamente. De 1.400 sujetos seleccionados al azar con ALT y GGT normales, 398 casos fueron excluidos por deterioro de las muestras evidenciado por un índice sérico elevado ${ }^{23}$. Esto se atribuyó al prolongado tiempo de almacenamiento (rango 167 a 526 días, media 346 días). En 11 casos hubo pérdida de datos. Así, fue posible analizar los niveles de bilirrubina en 991 individuos (37,3\% hombres). La Tabla 1 describe los datos generales de los sujetos evaluados. Seis coma cuatro por ciento de los individuos presentó niveles de BT $>1 \mathrm{mg} /$ dL y $2,0 \%$ niveles de BT $\geq 1,4 \mathrm{mg} / \mathrm{dl}$ (prevalencia cruda de SG). Los hombres presentaron con mayor frecuencia bilirrubinemia elevada al compararlos con mujeres ( $10,9 \%$ vs $3,9 \%$, respectivamente).

La Tabla 2 muestra la estimación de las prevalencias expandidas de SG según el muestreo complejo de la ENS, que es representativo de la población nacional chilena entre 15 y 64 años. La población expandida quedó compuesta por $44,9 \%$ de hombres, siendo levemente inferior a la población general chilena de acuerdo al censo 2012 (Población total 16.572.475, 48,6\% hombres) ${ }^{24}$. 
Así, la prevalencia poblacional expandida de SG en Chile fue de 2,6\%. El SG fue nueve veces más frecuente en hombres que en mujeres $(4,5 \%$ vs $0,5 \%)$. No se encontró correlación entre los niveles de bilirrubina y otras variables evaluadas en la ENS como edad, lugar de residencia, nivel educacional, tabaquismo, hipertensión arterial, colesterol total. Datos no presentados.

\section{Prevalencia poblacional de las variantes UGT1A1 28 y SLCO1B1c.T $>C$}

De las 500 muestras del estudio de La Florida ${ }^{21}$, $36,5 \%$ fueron hombres, con edad promedio de 51,1 años (rango 29 a 85 años). Fueron informativas 487 muestras para la variante UGT1A1 rs6742078 y 483 para la variante SLCO1B1 rs4149056. Como muestra la Tabla 3, la frecuencia de la variante infrecuente T del SNP rs6742078 del gen UGT1A1 fue de 31\% y genotipo homozigoto TT 11,7\% (genotipo Gilbert), similar a la frecuencia descrita en población alemana, española y europeas en general ${ }^{25,26}$ y significativamente mayor a la descrita en población china $(0,8 \%)^{27}$. La frecuencia del genotipo heterocigoto GT para esta variante en la cohorte chilena fue significativamente menor a la reportada en España y Alemania, pero mayor a la China. La frecuencia alélica de la variante infrecuente C del SNP rs4149056 del gen SLCO1B1 fue de 14,5\%, similar a lo descrito en Alemania y China. A su vez, la frecuencia el genotipo homocigoto CC fue de 3,1\%, similar a lo descrito en Alemania ${ }^{25}$ y mayor a la descrita en población china $^{28}$. Ambas variantes se encuentran en equilibrio de Hardy-Weinberg.

\section{Correlación fenotipo-genotipo}

Los tres grupos resultaron ser similares en edad, pero el grupo control quedó conformado por más mujeres comparado con el grupo intermedio y Gilbert (Tabla 4). El análisis de la variante UGT1A ${ }^{\star} 28$ demostró que la mayoría de los sujetos con SG (74\%) fueron homocigotos TA7/7. En contraste, el $44 \%$ de los sujetos con valores intermedios de BT y sólo 7\% de los controles fueron homocigotos TA7/7, existiendo una asociación significativa entre el genotipo y los niveles de bilirrubinemia (chi-cuadrado, $\mathrm{p}<0,0001$ ) (Tabla 5). Interesantemente, la presencia de uno o dos alelos TA7 se asocian a incrementos significativos de los niveles de bilirrubina sérica (Figura 1). El análisis de la variante UGT1A1 ${ }^{\star} 28$ por secuenciación directa estuvo en perfecto desequilibrio de unión ( $\left.\mathrm{LD}, \mathrm{r}^{2}=0,99\right)$ con el análisis de la variante rs6742078, siendo mayor a lo reportado en población caucásica $\left(\mathrm{r}^{2}=0,88\right)^{17}$.

El análisis de la variante SCLO1B1 c.T > C muestra que el genotipo CC es infrecuente, siendo similar en los 3 grupos (4\% en SG, $3 \%$ en intermedio y controles) y similar a la observada

Tabla 1. Características de los sujetos de la muestra ENS 2009-2010 analizada para la estimación de prevalencia de síndrome de Gilbert*

\begin{tabular}{|ll|}
\hline $\mathrm{n}$ seleccionado & 1.400 \\
\hline $\mathrm{n}$ evaluado & 991 \\
\hline Edad (años) & $38,7 \pm 14,2$ \\
\hline$\%$ Hombres & 37,3 \\
\hline $\begin{array}{l}\text { Bilirrubina total } \\
\text { Bilirrubina } \leq 1,0 \mathrm{mg} / \mathrm{dl} \\
\text { (\% del total) }\end{array}$ & $0,54 \pm 0,33 \mathrm{mg} / \mathrm{dL}$ \\
\hline $\begin{array}{l}\text { Bilirrubina }>1 \mathrm{y}<1,4 \mathrm{mg} / \mathrm{dl} \\
\text { (\% del total) }\end{array}$ & $93,5 \%$ \\
$\begin{array}{l}\text { Bilirrubina } \geq 1,4 \mathrm{mg} / \mathrm{dl} \\
\text { (\% del total) }\end{array}$ & $2,0 \%$ \\
\hline
\end{tabular}

*Se indica $n$ muestrales y parámetros crudos (no expandidos).

Tabla 2. Prevalencia fenotípica de Síndrome de Gilbert en Chile, ENS 2009-2010

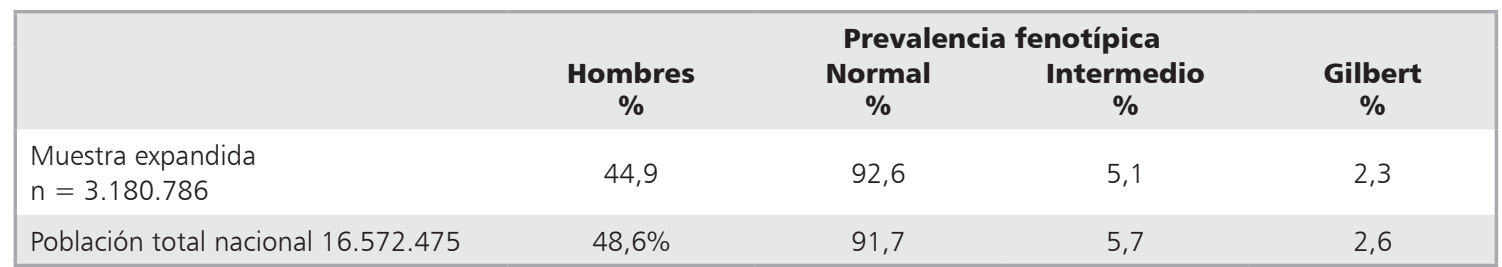

(*) Prevalencia fenotípica de SG de acuerdo a valores de bilirrubina sérica total (test colorimétrico HITACHI). Normal Bt $\leq 1 \mathrm{mg} /$ $\mathrm{dL}$, Intermedio Bt $>1 \mathrm{y}<1,4 \mathrm{mg} / \mathrm{dL}$, Gilbert Bt $\geq 1,4 \mathrm{mg} / \mathrm{dL}$. Sujetos con transaminasas normales. 
en la muestra de La Florida (3,1\%, Tabla 3). Las frecuencias de los genotipos TT y TC fue también similar entre los grupos estudiados. Dada esta baja capacidad discrimatoria de la variante SCLO1B1
c.T $>$ C, analizamos si la presencia una copia de alelo C (TC o CC) se distribuía de forma asimétrica entre los grupos, sin encontrar diferencias significativas $(\mathrm{p}=0,069)$.

Tabla 3. Prevalencia poblacional de las variantes UGT1A1 rs6742078 (G > T) y SLC01B1 rs4149056 $(\mathrm{T}>\mathrm{C}$ ) en población chilena comparada con población alemana, española y china

\begin{tabular}{|c|c|c|c|c|c|c|}
\hline \multicolumn{7}{|c|}{ UGT1A1 rs6742078 } \\
\hline País & $\mathbf{n}$ & $\begin{array}{c}\text { GG }(6 / 6) \\
n \quad(\%)\end{array}$ & $\begin{array}{c}\text { GT }(6 / 7) \\
n \quad(\%)\end{array}$ & $\begin{array}{c}\text { TT (7/ 7) } \\
\text { n } \quad(\%)\end{array}$ & $\begin{array}{c}\text { Frecuen } \\
\text { G (TA6) } \\
\%\end{array}$ & $\begin{array}{c}\text { a alélica } \\
\text { T (TA7) } \\
\%\end{array}$ \\
\hline Chile & 487 & $246(50,5)$ & $184(37,8)$ & $57(11,7)$ & 69 & 31 \\
\hline España $^{\dagger}$ & 100 & $40(40,0)^{(a)}$ & $51(51,0)^{(a)}$ & $9(9,0)^{(a)}$ & 65,5 & $34,5^{(a)}$ \\
\hline Alemania & 3.727 & $1.649(44,2)^{(a)}$ & $1.631(43,8)^{(a)}$ & $447(12,0)^{(a)}$ & 66 & $34^{(a)}$ \\
\hline China & 539 & $417(77,2)^{(b)}$ & $118(22,0)^{(b)}$ & $4(0,8)^{(b)}$ & 88 & $12^{(\mathrm{b})}$ \\
\hline Test Fisher & & $<0,05^{(a)},<0,001^{(b)}$ & $<0,05^{(a)},<0,001^{(b)}$ & $N S^{(a)},<0,001^{(b)}$ & $N S^{(a)},<$ & $0001^{(b)}$ \\
\hline
\end{tabular}

Test exacto de Fisher; ${ }^{(a)}$ Chile vs España o Alemania, ${ }^{(b)}$ Chile vs China; NS = no significativo. ${ }^{\dagger}$ Cohorte española de donantes de sangre, no poblacional.

\begin{tabular}{|c|c|c|c|c|c|c|}
\hline \multicolumn{7}{|c|}{ SLC01B1 rs4149056 } \\
\hline \multirow[t]{2}{*}{ País } & \multirow[t]{2}{*}{$\mathbf{n}$} & \multirow{2}{*}{$\begin{array}{cc}\text { TT } \\
\text { n } \quad(\%)\end{array}$} & \multirow{2}{*}{$\begin{array}{c}\text { TC } \\
\text { n }(\%)\end{array}$} & \multirow{2}{*}{$\begin{array}{c}\text { CC } \\
\text { n }(\%)\end{array}$} & \multicolumn{2}{|c|}{ Frecuencia alélica } \\
\hline & & & & & $\begin{array}{c}\mathbf{T} \\
\%\end{array}$ & $\begin{array}{l}\text { C } \\
\%\end{array}$ \\
\hline Chile & 483 & $358(74,1)$ & $110(22,8)$ & $15(3,1)$ & 85,5 & 14,5 \\
\hline Alemania & 3.641 & $2513(69,0)^{(a)}$ & $1026(28,2)^{(a)}$ & $102(2,8)^{(a)}$ & 83,1 & $16,9^{\text {(a) }}$ \\
\hline China & 100 & $75(75)^{(b)}$ & $24(24)^{(b)}$ & $1(1)^{(b)}$ & 87 & $13^{(b)}$ \\
\hline \multicolumn{2}{|c|}{ Test Fisher valor ( $p)$} & $<0,05^{(a)}, N S^{(b)}$ & $<0,05^{(\mathrm{a})}, \mathrm{NS}^{(\mathrm{b})}$ & $N S^{(a)},<0,05^{(b)}$ & \multicolumn{2}{|c|}{$N S^{(a)}, N S^{(b)}$} \\
\hline
\end{tabular}

Test exacto de Fisher; ${ }^{(a)}$ Chile vs Alemania, ${ }^{(b)}$ Chile vs China.

Tabla 4. Características basales de sujetos de estudio casos y controles agrupados según bilirrubina sérica

\begin{tabular}{|lcccc|}
\hline Fenotipo & $\mathbf{n}$ & Edad promedio & \% hombres & BT $\mathbf{~ m g / d L}$ \\
\hline Gilbert & 100 & 26,3 & 61 & $1,86 \pm 0,48^{*}$ \\
Intermedios & 100 & 26,8 & 57 & $1,18 \pm 0,11^{*}$ \\
Controles & 100 & 27,4 & $33^{* *}$ & $0,51 \pm 0,18^{*}$ \\
\hline
\end{tabular}

*Diferencia estadísticamente significativa para los 3 grupos, $p<0,05$. **Diferencia estadísticamente significativa respecto de grupo Gilbert e intermedio, $\mathrm{p}<0,05$.

Tabla 5. Frecuencia de los genotipos para las variantes UGT1A1*28 y SLCO1B1 en los tres grupos fenotípicos de acuerdo a bilirrubina sérica

\begin{tabular}{|c|c|c|c|c|c|c|c|}
\hline \multirow[t]{2}{*}{ Fenotipo } & \multirow[t]{2}{*}{ (n) } & \multicolumn{3}{|c|}{ UGT1A1*28 } & \multicolumn{3}{|c|}{ SLC01B1 rs4149056 } \\
\hline & & $6 / 6$ & $6 / 7$ & 7/7 & TT & CT & CC \\
\hline Gilbert & $(100)$ & 4 & 22 & 74 & 61 & 35 & 4 \\
\hline Intermedios & $(100)$ & 15 & 41 & 44 & 71 & 26 & 3 \\
\hline Controles & $(100)$ & 42 & 51 & 7 & 73 & 24 & 3 \\
\hline
\end{tabular}




\section{Discusión}

En el presente estudio evaluamos por primera vez en Chile la frecuencia poblacional de SG y sus principales determinantes genéticas.

Nuestros resultados confirman que en la población chilena el SG es una condición frecuente. De acuerdo a la definición utilizada, la prevalencia en población general $(2,6 \%)$ resultó ser discretamente inferior a la reportada en otras poblaciones utilizando el mismo punto de corte ${ }^{29}$. El diseño de la ENS2009-2010, con toma de muestras de sangre en regiones, procesamiento local inicial, posterior transporte con cadenas de frío y almacenamiento a $-20^{\circ} \mathrm{C}$ en Santiago, pudo ser un factor que afectó los valores de bilirrubina obtenidos. De hecho, un estudio reciente demostró que sueros almacenados a $-20^{\circ} \mathrm{C}$ muestran una disminución significativa en los valores de BT a partir de 2 semanas, comparados con determinaciones en muestras frescas. El almacenamiento a $-80^{\circ} \mathrm{C}$ disminuye este artefacto $^{30}$. En la ENS2009-2010, el lapso transcurrido entre la toma de muestra y la determinación de bilirrubina fue de 167 a 526 días. Por otro lado, una ventaja del diseño de la ENS es que las determinaciones bioquímicas se efectuaron en un solo centro con altos estándares de calidad. Si bien el estándar de oro para la determinación de bilirrubina es la cromatografía líquida de alta presión (HPLC), optamos por el método colorimétrico por su disponibilidad y bajo costo. Las técnicas colorimétricas han sido utilizadas en la mayoría de los estudios poblacionales ${ }^{29}$.

La bilirrubina sérica es uno de los parámetros de laboratorio con mayor variabilidad intra e inter individuo ${ }^{31,32}$. Se acepta un error máximo tolerable de $20-30 \%$ (rango $\pm 0,4 \mathrm{mg} / \mathrm{dL})^{33}$ y hasta el momento no existe una definición universalmente consensuada para el fenotipo $\mathrm{SG}^{5,29}$. El umbral de $\mathrm{BT} \geq 1,4 \mathrm{mg} / \mathrm{dL}$ para el diagnóstico de $\mathrm{SG}$, utilizado en estudios de prevalencia similares al nuestro ${ }^{5}$, es más específico pero puede disminuir la sensibilidad diagnóstica. Así, en la ENS 2009-2010 la prevalencia sería $7,4 \%$ si utilizaramos un valor $>1 \mathrm{mg} / \mathrm{dL}$ (Tabla 2). Por último, la medición de bilirrubina en forma seriada puede incrementar la sensibilidad diagnóstica de $\mathrm{SG}^{29}$.

La prevalencia poblacional fenotípica de SG presentó diferencias significativas entre hombres y mujeres (9 veces mayor en hombres), no atribuibles al genotipo, observación que ha sido documentada previamente ${ }^{34}$. Se postula que las hormonas sexuales femeninas ${ }^{9}$ pueden incrementar la conjugación y secreción hepática de bilirrubina. En modelos animales estas diferencias desaparecen con la gonadectomía9.

Demostramos que la mayor parte de los casos de SG $(\sim 75 \%)$, utilizando la definición fenotípica de BT $\geq 1,4 \mathrm{mg} / \mathrm{dl}$, es explicado por homocigocidad para el alelo UGT1A1²8. Es llamativo que $44 \%$ de sujetos con elevaciones intermedias de bilirrubina $(1-1,4 \mathrm{mg} / \mathrm{dL})$ fueron también homocigotos para el alelo UGT1A1 ${ }^{\star} 28$, en contraste con sólo $7 \%$ en los sujetos con bilirrubinemia normal. Esto sugiere que un porcentaje significativo de individuos con bilirrubina en valores intermedios e incluso normales, pueden ser portadores de SG. Estos fenotipos atenuados u ocultos de SG pudieran ser detectados con muestreo frecuente o en condiciones de desafío, como ayuno prolongado $^{35}$, infecciones, o algunos fármacos ${ }^{36,37}$. No se ha evaluado sistemáticamente si los individuos con genotipo TA7/TA7, se comportan fenotípicamente diferentes ante un desafío estandarizado, comparado a los sujetos TA6/TA7 o TA6/TA6 $6^{35}$. Interesantemente, la presencia de uno o más alelos TA7 se asociaron a niveles crecientes de bilirrubinemia (Figura 1), confirmando la relevancia de esta variante en modular los niveles de BT. Esto sugiere que la definición fenotípica histórica de SG requiere ser reconsiderada a la luz de las evidencias de la información de los determinantes genéticos.

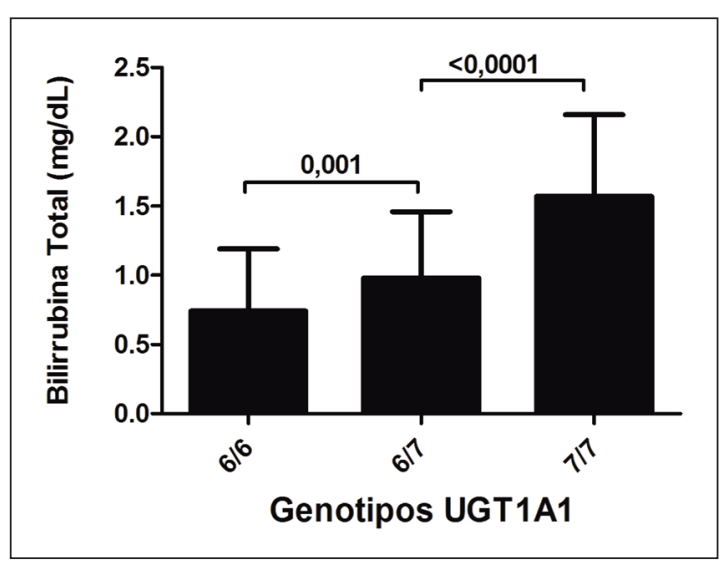

Figura 1. Correlación entre niveles plasmáticos de bilirrubina total y genotipo UGT1A1 en 300 individuos chilenos. La presencia de al menos un alelo infrecuente (heterocigotos 6/7) se asocia a un significativo mayor nivel de bilirrubina sérica. 
Síndrome de Gilbert en Chile - L. Méndez et al

Si bien la prevalencia poblacional fenotípica de SG resultó ser inferior a la reportada en poblaciones europeas, la frecuencia de la variante UGT1A1*28 resultó ser similar, lo que sugiere que otros factores (metodológicos, ambientales o genéticos) modifican la expresión fenotípica.

El SG dista mucho de ser sólo una condición "cosmética" o irrelevante. Estudios epidemiológicos han documentado una correlación inversa entre riesgo cardiovascular y oncológico (cáncer colorrectal) y los niveles de BT $\mathrm{BT}^{38,39,40}$. Estos hallazgos han sido replicados en diversos grupos étnicos ${ }^{41}$. Se postula que la bilirrubina ejerce un efecto protector antioxidante y/o antiinflamatorio $^{42}$. La bilirrubina tiene mayor capacidad en reducir la oxidación lipídica que el glutatión y la vitamina $\mathrm{E}^{43,44}$. Mayores niveles de BT se asocian con un menor riesgo de esteatohepatitis no-alcohólica (NASH) $)^{45}$, nefropatía diabética ${ }^{46}$ y reestenosis de stent coronario ${ }^{47}$. Se ha propuesto incluso que sería útil inducir artificialmente una hiperbilirrubinemia leve para prevención de estas enfermedades ${ }^{38}$. Dentro de los potenciales riesgos que implica ser portador de un genotipo SG, Buch y cols han demostrado que la variante UGT1A1 ${ }^{\star} 28$ constituye un factor de riesgo de colelitiasis en hombres ${ }^{25}$. Además se ha descrito que los individuos portadores de SG tienen mayor riesgo a toxicidad por fármacos como el irinotecan y algunos antirretrovirales, por lo que el fenotipo y genotipo SG puede considerarse como un factor de riesgo farmacogenético.

En síntesis, en el presente estudio demostramos que la prevalencia fenotípica de SG en la población chilena adulta es igual o superior a 2,6\%, siendo el genotipo de SG similar a poblaciones europeas. El fenotipo SG en Chile es explicado en gran medida por la variante UGT1A $1^{\star} 28$, al igual que en poblaciones europeas. Dada la relevancia de esta condición en diferentes escenarios clínicos de enfermedades prevalentes como en farmacogenética, el diagnóstico específico de SG combinando las herramientas de laboratorio clásicas con un análisis genético específico, es altamente deseable.

Agradecimientos: Los autores agradecen la valiosa colaboración de Carlos Salcedo, Ingeniero Informático, Jacqueline Parada y Ligia Valdivia, tecnólogos médicos, Servicios de Laboratorios Clínicos, Pontificia Universidad Católica de Chile.
Agradecemos también los valiosos comentarios y sugerencias del Dr. Arnaldo Foradori, Facultad de Medicina, Pontifica Universidad Católica de Chile.

\section{Referencias}

1. Fevery J. Bilirubin in clinical practice: a review. Liver Int 2008; 28: 592-605.

2. Gilbert A LP. La cholemie simple familiale. Semaine Medicale 1901; 21:241-3.

3. Meulengracht E. A review of chronic intermittent juvenile jaundice. QJM 1947; 16: 83-98.

4. Adachi Y, Nanno T, Yamamoto T. [Japanese clinical statistical data of patients with constitutional jaundice]. Nihon Rinsho 1992; 50 Suppl: 677-85.

5. Owens D, Evans J. Population studies on Gilbert's syndrome. J Med Genet 1975; 12: 152-6.

6. Radu P, Atsmon J. Gilbert's syndrome-clinical and pharmacological implications. Isr Med Assoc J 2001; 3: 593-8.

7. Zucker SD, Goessling W, Hoppin AG. Unconjugated Bilirubin Exhibits Spontaneous Diffusion through Model Lipid Bilayers and Native Hepatocyte Membranes. Journal of Biological Chemistry 1999; 274: 10852-62.

8. Keppler D. Cholestasis and the role of basolateral efflux pumps. Z Gastroenterol 2011; 49: 1553-7.

9. Muraca M, Fevery J. Influence of sex and sex steroids on bilirubin uridine diphosphate-glucuronosyltransferase activity of rat liver. Gastroenterology 1984; 87: 308-13.

10. Rigato I, Ostrow JD, Tiribelli C. Bilirubin and the risk of common non-hepatic diseases. Trends in molecular medicine 2005;11:277-283.

11. Fevery J, Blanckaert N, Heirwegh KP, Preaux AM, Berthelot P. Unconjugated bilirubin and an increased proportion of bilirubin monoconjugates in the bile of patients with Gilbert's syndrome and Crigler-Najjar disease. J Clin Invest 1977; 60: 970-9.

12. Black M, Billing BH. Hepatic Bilirubin UDP-Glucuronyl Transferase Activity in Liver Disease and Gilbert's Syndrome. New England Journal of Medicine 1969; 280: 1266-71.

13. Bosma PJ, Chowdhury JR, Bakker C, Gantla S, de Boer A, Oostra BA, et al. The genetic basis of the reduced expression of bilirubin UDP-glucuronosyltransferase 1 in Gilbert's syndrome. N Engl J Med 1995; 333: 1171-5.

14. Clementi M, Di Gianantonio E, Fabris L, Forabosco P, Strazzabosco M, Tenconi R, et al. Inheritance of hyperbilirubinemia: evidence for a major autosomal recessive gene. Dig Liver Dis 2007; 39: 351-5.

15. Rouits E, Boisdron-Celle M, Dumont A, Guerin O, Morel A, Gamelin E. Relevance of different UGT1A1 
polymorphisms in irinotecan-induced toxicity: a molecular and clinical study of 75 patients. Clin Cancer Res 2004; 10: 5151-9.

16. Shulman K, Cohen I, Barnett-Griness O, Kuten A, Gruber SB, Lejbkowicz F, Rennert G. Clinical implications of UGT1A1*28 genotype testing in colorectal cancer patients. Cancer 2011; 117: 3156-62.

17. Johnson AD, Kavousi M, Smith AV, Chen MH, Dehghan A, et al. Genome-wide association meta-analysis for total serum bilirubin levels. Hum Mol Genet 2009; 18: 270010.

18. Link E, Parish S, Armitage J, Bowman L, Heath S, Matsuda F, et al. SLCO1B1 variants and statin-induced myopathy-a genomewide study. N Engl J Med 2008; 359: 789-99.

19. Lee JS, Wang J, Martin M, Germer S, Kenwright A, Benayed R, et al. Genetic variation in UGT1A1 typical of Gilbert syndrome is associated with unconjugated hyperbilirubinemia in patients receiving tocilizumab. Pharmacogenetics and Genomics 2011; 21: 365-74 10.1097/FPC.0b013e32834592fe.

20. Universidad DdSPdlFdMdlP, Católica de Chile MdS, Gobierno de Chile. Encuesta Nacional de Salud ENS Chile 2009-2010, 2010.

21. Miquel JF, Covarrubias C, Villaroel L, Mingrone G, Greco AV, Puglielli L, et al. Genetic epidemiology of cholesterol cholelithiasis among Chilean Hispanics, Amerindians, and Maoris. Gastroenterology 1998; 115: 937-46.

22. Buch S, Schafmayer C, Volzke H, Becker C, Franke A, von Eller-Eberstein $\mathrm{H}$, et al. A genome-wide association scan identifies the hepatic cholesterol transporter ABCG8 as a susceptibility factor for human gallstone disease. Nat Genet 2007; 39: 995-9.

23. Vermeer HJ, Thomassen E, de Jonge N. Automated Processing of Serum Indices Used for Interference Detection by the Laboratory Information System. Clinical Chemistry 2005; 51: 244-7.

24. INE. Resultados Preliminares Censo de Población y Vivienda 2012. Instituto Nacional de Estadísticas, 2012.

25. Buch S, Schafmayer C, Volzke H, Seeger M, Miquel JF, Sookoian SC, et al. Loci from a genome-wide analysis of bilirubin levels are associated with gallstone risk and composition. Gastroenterology 2010; 139: 1942-1951 e2.

26. Fernandez Salazar JM, Remacha Sevilla A, del Rio Conde E, Baiget Bastus M. [Distribution of the A(TA)7TAA genotype associated with Gilbert syndrome in the Spanish population]. Med Clin (Barc) 2000; 115: 540-1.

27. Zhang A, Xing Q, Qin S, Du J, Wang L, Yu L, et al. Intraethnic differences in genetic variants of the UGT-glu- curonosyltransferase $1 \mathrm{~A} 1$ gene in Chinese populations. Pharmacogenomics J 2007; 7: 333-8.

28. Jada SR, Xiaochen S, Yan LY, Xiaoqiang X, Lal S, Zhou SF, et al. Pharmacogenetics of SLCO1B1: haplotypes, htSNPs and hepatic expression in three distinct Asian populations. Eur J Clin Pharmacol 2007; 63: 555-63.

29. Okolicsanyi L, Nassuato G, Muraca M, Orlando R, Iemmolo RM, Lirussi F, et al. Epidemiology of unconjugated hyperbilirubinemia: revisited. Semin Liver Dis 1988; 8: 179-82.

30. Amin SB, Ahlfors C. Effect of storage and freezing on unbound bilirubin measurement. Clin Chim Acta 2008; 396: 56-7.

31. Siest G HJ, Schiele F, Young DS. Interpretation of Clinical Laboratory Tests: Reference Values and Their Biological Variation. Foster City, CA: Biomedical Publications; 1985, 1985.

32. Heil W. EV. Reference Ranges for Adults and Children Pre-Analytical Considerations. . ROCHE Diagnostic, 2008.

33. Heins M, Heil W, Withold W. Storage of serum or whole blood samples? Effects of time and temperature on 22 serum analytes. Eur J Clin Chem Clin Biochem 1995; 33 : 231-8.

34. Bailey A, Robinson D, Dawson AM. Does gilbert's disease exist? The Lancet 1977; 309: 931-3.

35. Teich N, Lehmann I, Rosendahl J, Troltzsch M, Mossner J, Schiefke I. The inverse starving test is not a suitable provocation test for Gilbert's syndrome. BMC Res Notes 2008; $1: 35$.

36. Preisig D, Bircher J, Preisig R. [Positive diagnosis of Gilbert syndrome. Retrospective analysis of 59 cases with special reference to the nicotinic acid test]. Schweiz Med Wochenschr 1982; 112: 1122-9.

37. Murthy GD, Byron D, Shoemaker D, Visweswaraiah $\mathrm{H}$, Pasquale D. The utility of rifampin in diagnosing Gilbert's syndrome. Am J Gastroenterol 2001; 96: 11504.

38. Schwertner HA, Vitek L. Gilbert syndrome, UGT1A1*28 allele, and cardiovascular disease risk: possible protective effects and therapeutic applications of bilirubin. Atherosclerosis 2008; 198: 1-11.

39. Vítek L, Jirsa Jr M, Brodanová M, Kaláb M, Mareček Z, Danzig V, et al. Gilbert syndrome and ischemic heart disease: a protective effect of elevated bilirubin levels. Atherosclerosis 2002; 160: 449-56.

40. Zucker SD, Horn PS, Sherman KE. Serum bilirubin levels in the U.S. Population: Gender effect and inverse correlation with colorectal cancer. Hepatology 2004; 40: 827-35.

41. Chin HJ, Song YR, Kim HS, Park M, Yoon HJ, Na KY, 
et al. The Bilirubin Level is Negatively Correlated with the Incidence of Hypertension in Normotensive Korean Population. J Korean Med Sci 2009; 24: S50-6.

42. Yoshino S, Hamasaki S, Ishida S, Kataoka T, Yoshikawa A, Oketani N, et al. Relationship between bilirubin concentration, coronary endothelial function, and inflammatory stress in overweight patients. J Atheroscler Thromb 2011; 18: 403-12.

43. Vitek L. The role of bilirubin in diabetes, metabolic syndrome, and cardiovascular diseases. Frontiers in Pharmacology 2012; 3.

44. Tapan S, Karadurmus N, Dogru T, Ercin CN, Tasci I, Bilgi C, et al. Decreased small dense LDL levels in Gilbert's syndrome. Clin Biochem 2011; 44: 300-3.

45. Hjelkrem M, Morales A, Williams CD, Harrison SA. Unconjugated hyperbilirubinemia is inversely associated with non-alcoholic steatohepatitis (NASH). Aliment Pharmacol Ther 2012; 35: 1416-23.

46. Inoguchi T, Sasaki S, Kobayashi K, Takayanagi R, Yamada T. Relationship between Gilbert syndrome and prevalence of vascular complications in patients with diabetes. JAMA 2007; 298: 1398-400.

47. Kuwano T, Miura S, Shirai K, Ike A, Mori K, Shimizu T, et al. Serum levels of bilirubin as an independent predictor of coronary in-stent restenosis: a new look at an old molecule. J Atheroscler Thromb 2011; 18: 574-83. 